Review

\title{
Applications of Energy Psychology in Addressing the Psychological Roots of Illness
}

\author{
David Feinstein *
}

777 East Main Street, Ashland, OR 97520; E-Mail: david@innersource.net

* Correspondence: David Feinstein; E-Mail: david@innersource.net

Academic Editor: Peta Stapleton

Special Issue: Mind-Body Approaches that are Revolutionizing the Health Field

OBM Integrative and Complementary Medicine

2021, volume 6 , issue 2

doi:10.21926/obm.icm.2102014
Received: February 01, 2021

Accepted: March 31, 2021

Published: April 19, 2021

\begin{abstract}
The impact of psychological factors on illness is, in recent years, being biochemically mapped. The subspecialties of psychosomatic medicine, health psychology, psychoneuroimmunology, and integrative medicine work with mind and body in concert to promote health and healing. A specialized set of mind-body approaches, collectively called "energy psychology," is being utilized within these and related clinical frameworks to facilitate beneficial changes in the neurological underpinnings of (1) mental states that impede immune function, (2) emotional influences that contribute to illness, and (3) inner resources that promote healing. Combining practices from time-honored healing traditions, particularly acupressure, with concepts and techniques drawn from contemporary psychology, practitioners using one of the most popular variations of the approach teach patients to tap on a prescribed series of acupuncture points while repeating phrases that activate areas of the brain that are involved with the issue receiving attention. Preliminary evidence supports speculation, which is consistent with more than a hundred peer-reviewed clinical trials, that the procedure can send deactivating signals to areas of the limbic system that are in hyperarousal and can send activating signals to regions of the prefrontal cortex that support executive functions such as planning and managing stressful situations. Ways of utilizing the approach for addressing emotional and cognitive aspects of physical illness are discussed. While utilizing the best medical
\end{abstract}

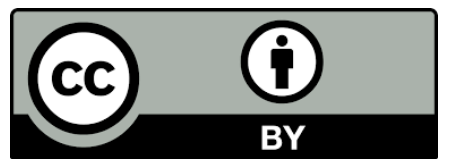

(c) 2021 by the author. This is an open access article distributed under the conditions of the Creative Commons by Attribution License, which permits unrestricted use, distribution, and reproduction in any medium or format, provided the original work is correctly cited. 
interventions available remains the first line of treatment, the potential value of working with the psychological aspects of disease is frequently underestimated. Energy psychology is proving to be a powerful tool for addressing this dimension of illness and healing. The paper closes with a detailed case history.

\section{Keywords}

Acupressure; anxiety; cognitive behavior therapy; emotional freedom techniques energy psychology; health; illness; tapping; thought field therapy

Body and soul respond to one another in sympathy.

-Aristotle, circa 400 B.C.

The physicians of ancient Greece believed that the life of the spirit is intertwined with the state of the body and that emotional balance is a primary factor in health [1]. Contemporary Western medicine has wrestled with just how to engage the mind and emotions in treating illness [2], a conundrum that traces back to Cartesian mind-body dualism. Many conventional as well as complementary medicine approaches have found their own ways for addressing this challenge [3]. This paper focuses on "energy psychology," a mind-body intervention that appears to have special strengths in its ability to rapidly focus on and impact psychological factors in illness.

\section{Background}

In 1936, Walter Cannon, then Chair of the Department of Physiology at Harvard, wrote "The Role of Emotion in Disease [4]," a paper that foreshadowed current understanding of the ways the endocrine, nervous, and immune systems communicate in advancing illness as well as in promoting health and healing. Cannon also coined the term "fight-or-flight response," advanced the concept of homeostasis, and popularized his theories in the book The Wisdom of the Body [5].

Franz Alexander built on Cannon's contributions in his classic text on psychosomatic medicine [6]. Published in 1950, it presented a compelling picture of the psychological factors involved in physical health and argued that these factors deserve the same detailed attention as physiological processes. The five fundamental principles of his approach included (1) the psychogenesis of illness, (2) physiological functions affected by psychological influences, (3) the specificity of emotional factors in somatic disturbances, (4) personality type and disease, and (5) the relation of nervous and hormonal mechanisms.

Cannon's and Alexander's contributions to the understanding of the role of psychological factors in illness and healing have become cornerstones not only of psychosomatic medicine [7], but also of health psychology [8], psychoneuroimmunology [9], and integrative medicine [10]. These specialties have demonstrated that mind and emotion are fundamentally entwined with diseasewhether in influencing its etiology, reacting to the challenges it presents, or catalyzing the healing process. Particularly important causal factors in illness related to psychological disposition and lifestyle include emotions [11], trauma [12], and stress [13]. 
A range of therapies that explicitly incorporate a somatic component actively utilize these principles. A comprehensive overview of body-mind approaches is presented in The Handbook of Body Psychotherapy and Somatic Psychology [14]. The practices covered date back to the work of Elsa Gindler and Wilhelm Reich and extend to modern iterations such as "somatic experiencing [15]" and "sensory-motor processing [16]." Each represents a unified body-mind perspective, based on the complementary premises that physical conditions affect mental health and mental conditions affect physical health. Energy psychology is one such approach.

\section{Energy Psychology}

Energy psychology is a hybrid discipline that has been shown to be particularly effective in rapidly altering maladaptive emotions and beliefs without a long course of psychotherapy [17]. It is referred to as a hybrid approach in that it combines practices from time-honored healing traditions, often including acupressure, with concepts and techniques drawn from contemporary psychology. For instance, in its most frequently applied format, acupuncture points on the face and body are manually stimulated while exposure techniques or cognitive interventions are being applied.

Rather than an independent modality, an energy psychology approach is most frequently incorporated into the practitioner's existing clinical framework [18]. The most popular formulations of the method are the Emotional Freedom Techniques (EFT) and Thought Field Therapy (TFT). Along with a number of other variations, the approach is sometimes referred to simply as "tapping." A standard protocol might involve having the patient tap on a sequence of around a dozen selected acupuncture points (acupoints) while a series of emotionally-evocative, personally-relevant phrases are spontaneously introduced by the patient or presented by the therapist and repeated by the patient.

The underlying principle is that the acupoint tapping sends activating or deactivating signals to specific brain areas that have been aroused by the phrases [19]. The stimulation of selected acupuncture points has been shown over the course of a ten-year research program at Harvard Medical School to rapidly reduce limbic system arousal [20, 21]. Deactivating signals can, for instance, counter the amygdala's threat response in real time while the patient is focusing on scenes that trigger irrational fears. Meanwhile, activating signals can enhance planning and stress management capacities governed by the prefrontal cortex. Tapping generates electric signals via the well-established principle of "mechanosensory transduction [22]." In this process, certain large proteins within the cells convert a mechanical stimulus, such as pressure on the skin, into electricity, known as piezoelectricity ("electricity generated by pressure"). Because the electrical impulses are apparently carried through the connective tissue by the semi-conductor collagen [23], they may reach the brain or other remote areas of the body undistorted and almost instantaneously, rather than traveling synapse to synapse through the nervous system. A 13-minute video illustrating an acupoint tapping session in the treatment of a height phobia provides a glimpse into the relatively unusual procedures (http://phobiacase.EnergyPsychEd.com, accessed March 24, 2021).

\subsection{Efficacy}

At the time of this writing, 115 clinical trials investigating the efficacy of energy psychology protocols have appeared in peer-reviewed journals and are listed in a database maintained by the
Association
for
Comprehensive
Energy
Psychology
(ACEP; 
www.energypsych.org/page/research_landing, accessed March 24, 2021). Of the 115 clinical trials, 113 reported statistically significant improvement in at least one of the targets for change being examined, often with unusual speed. Meta-analyses of subsets of these studies have documented efficacy for anxiety (14 studies, 658 participants) [24], depression (12 studies, 398 participants) [25], and post-traumatic stress disorder (7 studies, 247 participants) [26]. All three meta-analyses found large pre-treatment to post-treatment effect sizes across the studies reviewed (1.23 for anxiety; 1.85 for depression; 2.96 for PTSD.) Sizes above 0.8 indicate a large effect.

\subsection{Comparisons with Established Treatments}

Cognitive Behavior Therapy (CBT) and its variations are considered the "gold standard" for treating many psychological conditions [27]. Ten studies have been conducted which compare CBT with energy psychology protocols. An analysis of the outcomes found that acupoint tapping protocols performed at least equivalently to CBT in all ten studies, and nine of the ten comparisons showed some advantages of acupoint tapping protocols over CBT on measures such as speed, impact, or stability of gains at follow-up [19]. Two studies by independent research teams have compared a number of the most widely used trauma treatments (eg, CBT, Narrative Exposure Therapy, Eye Movement Desensitization and Reprocessing, play therapy, family therapy, and meditation) and found the energy psychology interventions to have produced among the largest effect sizes and greatest durability of benefits $[28,29]$.

\subsection{Speed}

Three head-to-head studies showed acupoint tapping to bring about at least equivalent improvements in fewer sessions than CBT [30-32]. Investigations of outcomes following a single energy psychology session of 30 to 60 minutes have shown statistically significant or substantial client-reported improvement in the fear of small animals [33, 34], claustrophobia [35], other specific phobias [36], insomnia [37], and the symptoms of frozen shoulder [38]. More sessions are of course required for more complex conditions, yet the average duration of treatment for a particular condition is usually shorter than expected. For instance, a randomized controlled trial (RCT) of the use of an acupoint tapping protocol for PTSD within a public health facility in Scotland allowed subjects to receive up to eight treatment sessions [39]. Voluntary termination of treatment occurred, however, after an average of 3.8 sessions, with a large overall effect size (1.0) on posttreatment measures and sustained gains on follow-up.

Another RCT, this one in the treatment of veterans with PTSD, had a low dropout rate and found that only $14 \%$ of 49 treated still had the disorder after six one-hour tapping sessions [40], a study that has been replicated with similar findings [41]. By way of contrast, CBT and its variations, which are the standards of care for treating PTSD [42], average 12 to 16 treatment sessions [43], and approximately two thirds of service members and veterans completing a course of CBT or its variations still met PTSD diagnostic criteria after treatment [44]. A 10-minute video illustrating an acupoint tapping approach in the rapid treatment of four combat veterans suffering with PTSD can be viewed at http://www.vetcases.com, accessed March 24, 2021).

\subsection{Durability}


Of the 115 clinical trials in the ACEP database, 79 did follow-up, with 77 finding that benefits were sustained. Follow-up periods ranged from one month to two years, with a mean of 7.0 months. "Benefits were sustained" was defined as meaning that follow-up testing showed a statistically significant $(p<.05)$ improvement between pre-treatment assessments and assessments at the end of the follow-up period on at least one of the major targets for change being tracked [19].

\subsection{Conditions That Respond}

Psychological conditions in the ACEP database that have responded to acupoint tapping treatments include anxiety, depression, phobias, PTSD, anger, stress, concentration difficulties, food cravings, insomnia, and performance blocks. Physical conditions in the ACEP database, which are not typically treated with psychotherapy but have shown improvement after energy psychology protocols include psoriasis, fibromyalgia, headaches, frozen shoulder, obesity, pulmonary injuries, immune function, cardiovascular function, chemotherapy side effects, and seizure disorders. Therapies that are able to treat a wide range of disorders are presumably impacting a smaller set of underlying causes. Acupoint tapping protocols have been shown to facilitate shifts in gene expression associated with improved health and mental health $[45,46]$, reduce the production of stress hormones [47, 48], normalize brainwave patterns [49], and disrupt the neurological structure of stimulus-response relationships [50].

\subsection{Association with Acupuncture}

Because of its reliance on acupuncture points, the credibility of energy psychology is tied to the status of acupuncture. At least 361 points on the surface of the skin are recognized in Traditional Chinese Medicine as having special properties compared to neighboring areas of the skin [51]. These physiological properties include higher electrical conductivity and a corresponding lower electrical resistance [52, 53], helping explain the effect of acupuncture on the body's electrochemical activity. While the reception of acupuncture in the West has been mixed, a definitive review of more than a thousand peer-reviewed RCTs is both comprehensive and rigorous, identifying "moderate" to "high quality" evidence of beneficial effects with 46 conditions, including asthma, hypertension, irritable bowel syndrome, osteoarthritis, postoperative nausea, constipation, stroke rehabilitation, anxiety, insomnia, and various types of pain [54]. Acupuncture and energy psychology are vastly different practices, but traditional needling on an acupoint and stimulating it manually with tapping generate similar effects. A double-blind study comparing penetration by acupuncture needles with nonpenetrating pressure found equivalent clinical improvements for each intervention [55]. While each approach has its place in health care, a distinct advantage of tapping protocols over needling for working with emotional conditions is, in addition to not requiring special equipment and extensive training, in the way the practitioner can adjust the treatment strategy from moment to moment.

\section{Addressing the Emotional Components of Illness}

Three interrelated ways that energy psychology protocols can be applied in medical settings include (1) managing mental states that impede immune function and healing, (2) working with emotional influences that contribute to the illness, and (3) mobilizing inner resources that promote healing. Each will be addressed separately. 


\subsection{Managing Mental States That Impede Immune Function and Healing}

Predictable responses to a serious medical diagnosis include fear (which is focused on specific anticipations) or a more generalized anxiety, along with uncertainty, insecurity, and disorientation. Meanwhile, denial that is fueled by fear is a coping mechanism that gives a person time to adjust to a distressing diagnosis. While denial serves this useful function and should not be confronted without sensitivity, it can also interfere with treatment as well as with taking the necessary backhome steps for supporting the healing process, such as recommended changes in diet, rest, and lifestyle.

\subsubsection{Embracing Both Sides of Internal Conflict}

When encountering such an internal conflict, the general approach within energy psychology is to acknowledge, accept, and recognize the positive intention of each side of the conflict. After carefully listening to the patient's description of thoughts and feelings that have arisen since the diagnosis, a phrase that could be paired with the acupoint tapping might be "Even though I hear the diagnosis, I don't want to believe it." Such wording validates, in this case, the external reality that the patient is navigating as well as the contradictory emotional reality it has generated. The conflict itself may become a source of internal distress. The acupoint stimulation sends deactivating signals to the limbic system [20, 21], which reduces the distress. This allows the amygdala and the hippocampus to process the contrasting realities contained within the dilemma in a more coherent manner, ultimately hastening the person's ability to take the next psychological steps in confronting the illness.

Such internal conflicts, called "psychological reversals [56]," often result in the reverse of what the person is intending. If the underlying desire to not believe the diagnosis goes unattended, it may undermine the person's conscious intention to mobilize for addressing the illness. Additional psychological reversals when dealing with an illness might include feelings of not deserving to heal, a belief that healing is not possible, or a "secondary gain" such as sympathy the illness may have been bringing. Tapping on statements that acknowledge both sides of a psychological reversal seems to shift the way in which the conflict is psychologically configured. For instance: "Even though I love being taken care of, I want to get better." Both sides can be put into a single statement in that basic format, or such conflicting statements might alternate with subsequent tapping points: "I don't have to cook as long as I'm sick." "I long to be independent again." "Part of me doesn't want to go back to work." "I'm supporting my body as it heals." While different brain mechanisms are activated by different types of statements, the guiding assumption is that the body is geared toward healing and the tapping statements are processed within that context.

\subsubsection{Managing Challenging Emotions}

Other emotions such as anger, depression, hopelessness, shame, or grief may also become involved following a serious diagnosis. The adverse impact of anxiety and depression on health and immune function is well-established [57, 58], with lower lymphocyte counts and other chemical measures of a compromised immune response having been identified [59]. The meta-analyses cited earlier, showing strong effect sizes when energy psychology was used in treating anxiety [24], depression [25], and post-traumatic stress [26], are particularly germane when applying energy 
psychology protocols to illness. In addition to emotions about the illness itself, an exacting challenge involves the natural anxiety and fear around invasive procedures that are often an element of medical treatment. Energy psychology protocols have been shown to be effective in reducing fear and anxiety about medical [60] as well as dental [61] procedures.

\subsubsection{Sending Downregulating Signals to Activated Brain Areas}

A neurological mechanism underlying these decreases in the intensity of problematic emotional responses was demonstrated in the Harvard research program investigating the effects of working with various acupuncture points [21, 22]. Imaging equipment, including fMRI (functional magnetic resonance imaging) and PET (positron emission tomography) scans, showed that stimulating specific acupoints sends signals to the amygdala and other areas of the limbic system that rapidly reduce arousal.

This finding explains a key dynamic in applying acupoint tapping for physical healing. Clinical trials show that maladaptive physical, emotional, and behavioral reactions to triggers such as a traumatic memory or a sense of tightness in one's throat can be interrupted with energy psychology protocols. A comparison of pre- and post-treatment fMRI scans is illustrative, showing how arousal in brain areas associated with the condition being treated was eliminated (Figure 1). The study investigated outcomes in a program for managing food cravings in individuals being treated for obesity [50]. While no single area of the brain governs the complex responses to food, fMRI scans while images of high-caloric food were being presented showed activation in the superior temporal gyrus (associated with cognition) and the lateral orbito-frontal cortex (associated with reward). Posttreatment scans while the same images were displayed in random sequences showed substantially less or even no activation in these areas.

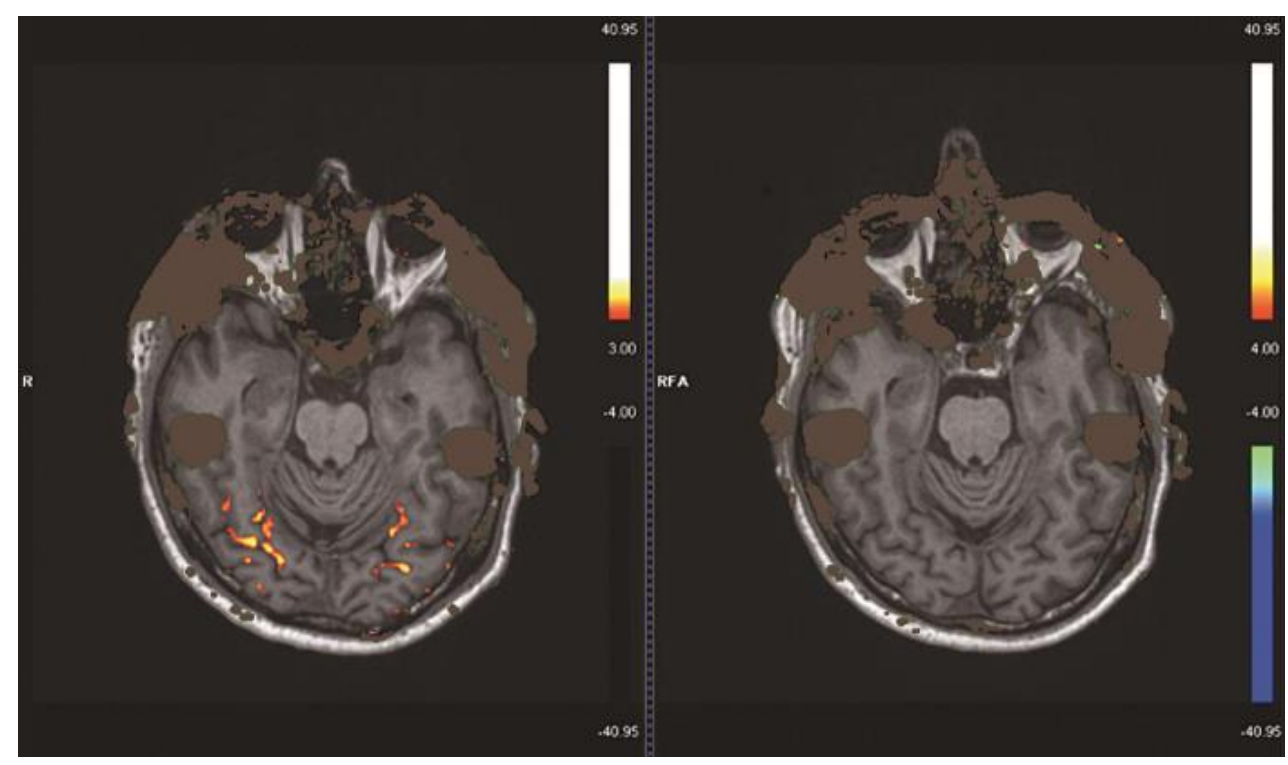

Figure 1 Pre- and post-treatment fMRI scans for subject in the tapping treatment of food cravings while high-caloric food images were presented. Colored areas activated during the pre-scan were not activated during the post-scan. From Stapleton et al, 2019 [50]. 
The decreased brain activation corresponded with a diminished desire for the targeted foods. Similar changes in neurological activation presumably occur in the clinical trials showing significant reductions in anxiety, depression, and PTSD discussed earlier [24-26].

\subsubsection{Triggering Biological Shifts That Support Healing}

Helping patients better manage difficult emotions generated by their illness not only leads to an ability to cope with the disease more effectively. It also supports the healing process at the biochemical level. In a pioneering study by Church et al. [47], a single one-hour energy psychology session was significantly more effective in lowering cortisol levels than a supportive counseling session, a finding that was recently replicated [48]. Acupoint tapping treatments were also shown to alter, in clinically desirable directions, the expression of genes that influence immunity, inflammation, and stress response $[45,46]$. An investigation of positive changes in physiological markers of health following acupoint tapping sessions found that decreases in blood pressure, resting heart rate, and cortisol levels corresponded with reductions in anxiety, depression, PTSD, chronic pain, and cravings [62].

\subsubsection{Addressing Subconscious Processes}

Most bodily processes occur at a subconscious or unconscious level. You do not have to tell your heart to beat, your stomach to digest, or your hair to grow. You can, however, consciously participate with many physiological activities. You can slow your breathing or relax your muscles. But can you send "healing energy" to a wound? Does a mother kissing her child's cut finger assist the repair process? Can you send messages to the body that impact illness and enhance healing? Energy psychology practitioners often use the wordings that accompany the tapping to attempt just that. Preparing for a surgery, the patient might tap on wordings such as:

Dear body, I want you to know that the surgery that is going to happen might feel like an assault.

But it is only happening to help and heal us.

It is not cruel or mean-spirted.

The surgeon is our friend, not an enemy.

Knowing this, you can prepare to meet the surgery in the most positive way possible.

Lots of people have been helped by this operation.

You don't need to fight it.

You don't need to mobilize against the invasion.

You can put all your energy into healing.

I am allowing this to happen for our mutual benefit.

I am sorry for the invasion. I don't want you to hurt.

I am going to do everything I can to bring you comfort.

Whether these self-suggestions, amplified by the tapping, actually impact the body's processes is a matter for future research, but that they give the person greater confidence and a more positive attitude about the medical procedure is supported by clinical experience [63] and patient reports [64]. 


\subsubsection{Back-Home Applications}

An energy psychology approach can also be applied on a back-home basis. Many practitioners give their patients "homework" to maintain their gains and encourage their engagement with the method. Energy psychology protocols have been successfully applied in the self-management of stress [31], anxiety [24], and symptoms of fibromyalgia [65]. A mobile app that guides users in applying acupoint tapping protocols for anxiety and stress was investigated in a large-scale study including 270,461 app users and found highly significant $(p<0.001)$ symptom reduction [66].

\subsection{Working with Emotional Influences That Contribute to the IIIness}

Energy psychology protocols, by their nature, focus on the interplay of cognition, emotions, and somatic events and experiences. This somatic element affords them an advantage in exploring the roots of illness. Working at this level often uncovers deeper psychological issues that may have been involved in the genesis of the illness itself. Symptoms such as pain, tension, and physical discomfort are somatic signals that can not only be regulated with acupoint tapping; they can serve as windows into the patient's history.

\subsubsection{Roots of Illness in Early Experiences}

Research connecting childhood experiences with illnesses in adult life has demonstrated that unresolved losses, traumas, or abuse reaching back to childhood are often pre-existing conditions in the etiology of illness [67]. The seminal Adverse Childhood Events (ACEs) study found that incidents of violence, emotional abuse, or family drug abuse consistently result in a variety of vulnerabilities [68]. The greater the number of ACEs in childhood, the greater the likelihood of chronic physical illnesses or psychological dysfunction in adulthood. Applying acupoint tapping protocols to unresolved emotional incidents downregulates limbic arousal, expanding therapeutic access to self-limiting cognitive and behavioral patterns that were organized around the emotional injuries.

\subsubsection{Questions That Evoke a Deeper Understanding}

Simply asking the person, "What was happening in your life when your symptoms first appeared" may be revealing. Answers such as "That was right after my mother died" or "I had lost my job and my marriage was breaking up" are not unusual. Tapping while keeping these memories mentally active seems to reduce their emotional power as obstacles to overcoming the illness.

Other questions that may provide entry into the psychological underpinnings of a patient's illness might include:

"If an emotion were associated with this illness, what might it be?"

"If a belief contributes to this illness, what might it be?"

"What are these symptoms telling you about your life?"

"If these symptoms reminded you of something in your family, what might that be?"

The responses to such questions can then be utilized in formulating the wordings that will accompany subsequent rounds of tapping, wherein the practitioner guides the patient to 
acknowledge, attune to, and tap on pertinent cognitions, emotions, images, and sensations that arise.

\subsubsection{Tapping on Underlying Issues}

A phenomenological analysis of practitioner interviews focusing on physical illness suggested that when predisposing events were identified, their emotional resolution via tapping protocols seemed to catalyze shifts that were believed to promote the healing process [63]. Systematic interviews with eight individuals receiving energy psychology treatments for help with physical illness revealed several themes that were able to be addressed by acupoint tapping. These included a) the role of unresolved emotional issues in the illness, b) outdated beliefs and self-defeating behavioral patterns that were contributing factors in the genesis and continuation of the illness, and c) ways the illness served as a buffer from the expectations of others [64]. All eight participants came to recognize that unresolved emotional issues played a role in their illness. A woman who had suffered with arthritis for years described this experience:

"My husband passed away almost 20 years ago. My children were really young at that time. So, there was really no time to stop and feel grief. And realizing that it was all those emotions that had been locked up. The lower back pain ... was gone in like one major session that we did. The pain was gone. It was like literally gone [laughs]. It was emotions and attachment that was holding in the back. Overnight when it cleared, I was like, 'Oh my God!' you know, 'it's just gone!'” [64].

A woman with chronic fatigue syndrome reflected that even though she was "doing all the things I was meant to be doing" for her health, such as eating well, meditation, et cetera, working with EFT brought her awareness to a different level:

"I realized that it could be underlying things that were keeping me unwell. So, it made me start to really look at what I had been through in my life and how I could resolve those traumatic events and those habits and programs I was running and my beliefs and things like that. And it made me realize that it was all that really had made me unwell. And it was all that, that was going to help me get well" [64].

\subsubsection{Outdated Patterns of Thoughts and Behavior}

Along with unresolved emotional issues from the past, some of those interviewed came to recognize how outdated beliefs and self-defeating behavioral patterns were contributing to their illness. A woman diagnosed with mast cell activation disorder tended to push herself to exhaustion. She reflected that her "work in EFT has been not doing that . . I'm now picking my battles ... simplifying my life and doing what really matters to me" [64]. A woman suffering with fibromyalgia and chronic migraines described how working with EFT helped bring these dynamics to the forefront so she could address them:

"Both my parents were war veterans ... there was alcoholism in the family, lots of violence. So, I learnt that I need to be independent. And I have to look after myself. Don't complain you know, when you are sick. You have to find your own way. Don't tell people. And don't give yourself that much attention. Just keep going. Just keep going, keep working . . . Be the good girl. . . And, rather than relaxing and taking it easy, telling people I am sick, and I can't come to work, when I started feeling the symptoms that day, I was like, "no you are going to work." You know it was the physical 
exhaustion. . . It was the body asking, like the body sending me signals. It calls you to attention. It just stops you" [64].

\subsubsection{Personal Relationships and Illness}

Another theme found in the interviews was the realization that illness can be a buffer from the expectations of other people. One of the participants observed that "a big shift" occurred when she realized that while she "had never been good at saying no," with the illness "I didn't have to do things I didn't want to" [67]. Another woman, who grew up "to be giving, giving to everybody," realized that her illness "was the only way my body knew . . . to have boundaries" [64]. A woman suffering with Crohn's Disease described herself as an "empath . . . I feel other people's pain . . . $85 \%$ of what I feel physically in my body is not mine." During the EFT sessions, she realized "If the Crohn's goes, I will have no excuse to hide" [64]. Discovering such underlying relationships and tapping on the experiences and emotions that maintain them can be a key to addressing psychological factors that are involved in physical illness.

\subsubsection{Anecdotal Reports}

In addition to these systematic interviews, hundreds of reports describing the experiences of people who have benefitted from tapping in working with a range of issues are posted on the internet (two sites which include such reports are http://www.EFTUniverse.com and http://www.emofree.com). Such anecdotal reports, of course, do not stand up as scientific evidence. Besides being entirely subjective, the number of people who used the technique and were not helped but didn't report it ("publication bias") is unknown. Such accounts are nonetheless still instructive for understanding the complex processes involved in the successful use of the technique.

Research on the course of illness after addressing emotional precursors using energy psychology protocols is still preliminary. On the clinical side, however, marked physical improvements occur often enough after focusing on these precursors that staying alert for such events in the history of a patient facing a serious disease is standard among tapping practitioners [63]. Sometimes the emotions around an early trauma can be processed relatively quickly with tapping protocols. In other instances, a longer term of psychotherapy may be required. The extensive case report presented later illustrates the interplay of the physiological and psychological factors involved in disease during tapping treatments.

\subsection{Mobilizing Inner Resources That Promote Healing}

Whatever the medical treatment, a variety of back-home behaviors can support it, such as wholesome nutrition, adequate sleep, regular exercise, and a positive attitude. Energy psychology protocols that successfully help build desired habits begin by focusing on obstacles to releasing the hold of habits that need to be replaced. Figure 1 showed how cravings for junk foods could be subdued. Tapping while bringing to mind scenarios that exemplify an obstacle to changing an undesirable mental habit or behavior pattern sends deactivating signals to the areas of the brain that are maintaining the pattern. This decreases the emotional charge on the objections to changing the habit, clearing the way for visualizing situations that might have triggered the old habit but instead result in an enactment of the desired response. For instance, imagine the recurring choice 
point at the end of a day of work at the computer screen. You have had dinner. Your body is restless. You could put on the news or do a 20-minute yoga routine you have previously learned. Visualizing yourself choosing the yoga routine, making statements about how much you are enjoying it, and sensing the good feelings after the stretching, all while tapping, seems to embed the imagery, sensations, and the statements' meanings into the nervous system, much like hypnotic suggestions, but more powerfully than imagery and verbal affirmations alone.

\subsubsection{Sedating and Activating Signals}

In a study of an acupoint tapping session that successfully eliminated a flying phobia [69], neural imaging showed that, as would be expected, the treatment downregulated activity in neural regions involved with the fear. But it also increased activity in frontal executive regions that are involved with rational choices and the management of emotional responses in stressful situations. Another fMRI study, conducted at Hannover Medical School in Germany, tracked 17 healthy subjects who stimulated acupoints while viewing fear-inducing and disgust-inducing images. The intensity of their subjective responses to the negative emotional stimuli was reduced. Activation in various brain regions involved in affect and information processing was selectively increased or decreased as required for adaptively "re-organizing the underlying neural pathways [70]."

Acupuncture points that decrease arousal are called "sedating points [71]." Sedating points send deactivating signals to specific areas of the brain or body, as demonstrated in the food craving study [50] and the Harvard brain imaging studies [20,21]. Acupoints that increase arousal are called "strengthening points [71]." Strengthening points send activating signals, as suggested by the flying phobia study [69]. Tapping on strengthening points while stating carefully formulated positive intentions seems to activate the growth of new neural pathways that can support the intention.

\subsubsection{Creating New Neural Pathways}

The images in Figure 2, from a scanning electron microscope, illustrate Donald Hebb's famous maxim that "neurons that fire together wire together." On the left, in the circle, you see the dendrite "tentacles" a distance apart from one another. In the middle, they are reaching toward each other, and on the right, they have connected. They have become wired together. This sequence can occur in as little as 12 seconds from the beginning of stimulation to the full connection of the dendrites [72]. Tapping on strengthening points while stating an intention or visualizing an outcome helps embed it into the brain's neural wiring. 

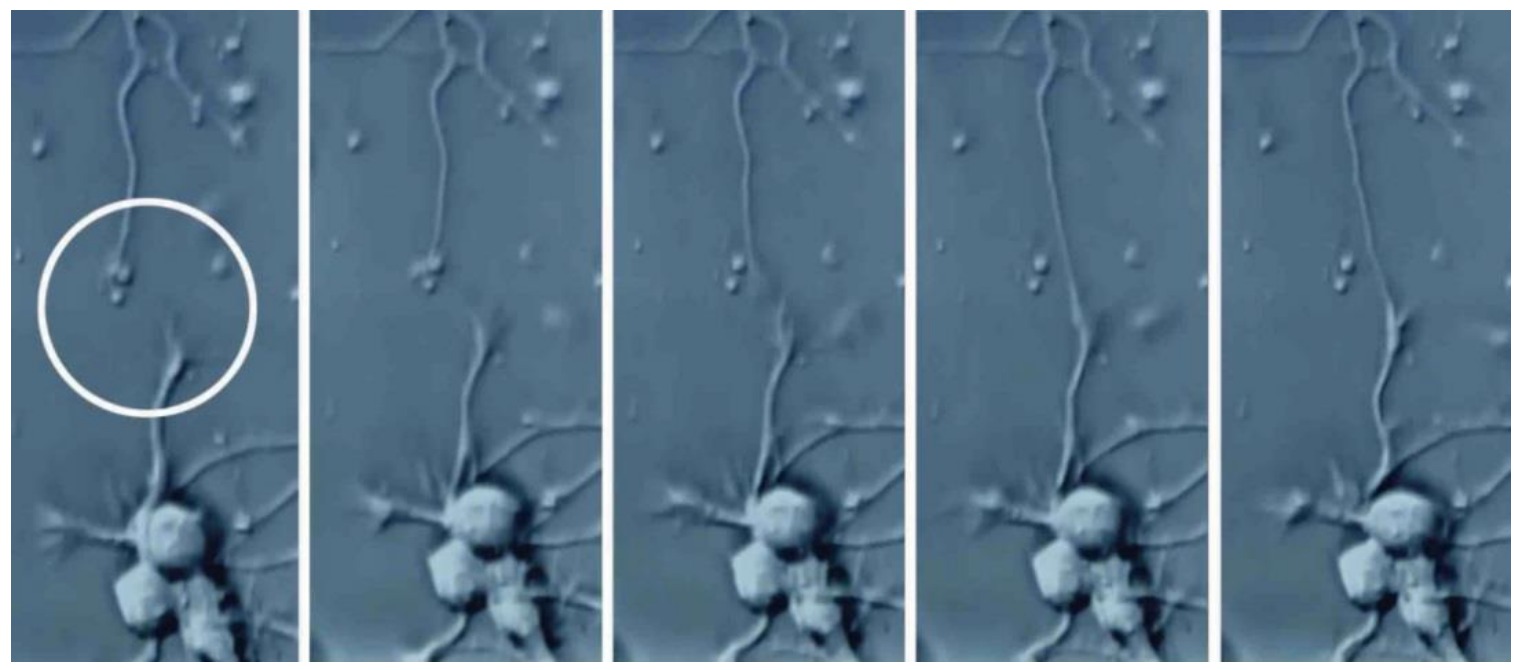

Figure 2 Neurons "wiring together" (from left to right) as seen through a scanning electronic microscope. From Church, 2020 [72].

The use of acupoint tapping to enhance the formation of new neural pathways may be applied in a variety of ways that extend beyond setting intentions for more positive behavioral habits. For instance, the best likely outcomes of the treatment may be visualized. While this is desired imagery, the process often begins with a triggering of inner doubts and objections. Their disempowering influence is, in the best outcomes, disarmed with tapping until a realistic and persistent positive image can be maintained. A statement that accompanies the acupoint stimulation might be: "Even though I am having difficulty believing I can get better, I am determined to find my way back to health." Visualizing a positive outcome while tapping associates, reinforces, and re-wires related beliefs, emotions, and behaviors-and perhaps even immune responses and other cellular activitiesthat are in alignment with the image.

\section{Clinical Evidence that Tapping is Effective in Addressing Physical Illness}

Systematic clinical trials have shown that energy psychology treatments have assisted patients with a variety of illnesses to adapt more effectively, including fibromyalgia [65], pulmonary injuries [73], side effects of chemotherapy [74], tension headaches [75], and blood sugar control in diabetes [76]. Case reports posted on https://eftuniverse.com/faqs/eft-and-tapping-hundreds-of-casestudies (accessed March 24, 2021) illustrate how acupoint tapping protocols have been helpful with Alzheimer's, cancer, chronic obstructive pulmonary disease, Crohn's, diabetes, Hodgkin's, Huntington's, Lyme's, Parkinson's, dermatological problems, and gastrointestinal disorders, among other medical conditions. The treatments described produced modest to major improvements in emotional difficulties and often physical symptoms as well. To offer a better feel for the procedure, a detailed summary of one of the more dramatic of the posted cases, with commentary, follows.

\subsection{Case Example}

Pauline had been diagnosed with Hodgkin's disease and a brain tumor three years before seeking energy psychology sessions from EFT practitioner Pascale Lutz. Chemotherapy to reduce Pauline's brain tumor was proving ineffective. At 38, with two young children, she had been told to "put her 
affairs in order." Over the next 10 months, Pauline had 20 sessions with Lutz. Fourteen of them took place during the first two months of their work together. Because the choice of wording is part of the art of delivery in energy psychology sessions [77], the wordings that accompanied some of the tapping during the initial sessions are presented.

In the first session, Pauline described intense pain in her right hand, arm, shoulder, and leg. Her right hand was shaking and had been frozen in a claw-like position for the previous two weeks. Pain reduction is a well established outcome for both acupuncture [54, 78], and energy psychology [38, $79,80]$, and the first round of tapping focused on the pain. Pauline rated its intensity as a 10 on the 0 to 10 Subjective Units of Distress (SUD) scale [81] that provides a quick assessment and is in common use in clinical settings. Some of the wordings Lutz had Pauline used during the first round of self-administered tapping on acupoints included:

Even though I have this pain in my right side that cuts like a knife, I deeply and completely love and accept myself. [As emphasized earlier, wording that pairs a symptom, problem, or difficulty with self-acceptance is a mainstay of energy psychology protocols. The tapping was accompanied by phrases such as:]

This pain like a knife in my right side.

This horrible pain.

This pain like a knife.

I'm so sick of it.

This pain.

The pain went down to 7. After another round of tapping on the remaining pain, it was down to 0 . Lutz inquired whether any uncomfortable sensations remained. Pauline reported, "It's like pins and needles." After another round of tapping, these were also gone. Then Pauline looked at her hand with amazement. The uncontrollable shaking had stopped, and her hand had completely loosened. For the first time in two weeks, Pauline was able to flex her fingers with ease, and her hand stayed steady. Lutz speculated that the blood recirculating was the source of the "pins and needles" sensation.

The second session was scheduled for six days later. A lumbar puncture four days before that session left Pauline with a constant headache and sore back. It was difficult to move in ways that stretched her back, and it was too painful to touch, despite morphine that had been prescribed following the procedure. The session first focused on her back condition, which Pauline described as a "red throbbing pain" and rated at 7. Among the wordings used during the first round of tapping:

Even though I have this red throbbing pain in my back due to the spinal tap, I deeply and completely love and accept myself.

This throbbing red pain in my back.

Etc.

The pain decreased to a 5, and Pauline now saw it as having changed color from red to orange:

Even though I still have this orange throbbing pain in my back, I deeply and completely love and accept myself.

This remaining orange throbbing pain. 
I don't need it in my life anymore. [Combining such statements with the acupoint tapping is believed to be more powerful than self-suggestion alone].

I have enough on my plate.

I choose to let it go with love.

Lutz next introduced a technique that is designed to shift the person's awareness, relax the body, and begin to integrate the experiences from the previous round of tapping. At this point Lutz noticed that Pauline's legs were shaking. On inquiry, Pauline said: "The pain is leaving my body. It's going down my legs and out my toes!" Lutz had Pauline resume the tapping, using wording intended to help her continue to let go of the pain "and everything bad with it." After a few minutes, the shaking subsided, and Pauline said that the pain was completely gone. She was then able to bend forward and backward, stretch, touch her back, and even press at the site of the puncture.

The focus then shifted to Pauline's headache, which she rated as an 8 in its intensity. She described it as an angry, agitated pain. After a round of tapping, the front of her head was clear. During the next round, the pain "left through my fingertips" while she was tapping. It went down to 0.

After the headache had subsided, Lutz asked Pauline how she visualized her cancer. Because a round of tapping can often be completed within a minute or so, multiple concerns may be addressed in a single session. Pauline said that the cancer looked like black smoke inside her body. Some relevant background about the next round of tapping: While Pauline's cancer had been diagnosed three years earlier, her oncologist estimated that its onset was approximately three years prior to diagnosis. Lutz asked what was happening in Pauline's life six years earlier. Pauline reflected that she had been pregnant and that her mother had died from cancer during her pregnancy. Pauline said she felt guilty about having a life growing inside her while her mother was dying. Wording in the next round included:

Even though I visualize my cancer like black smoke in my body, I now choose to release it.

This black smoke inside my body.

It's invading my body.

Since I knew my Mum was diagnosed with cancer.

This black smoke invaded my body.

It was the shock and the pain and the guilt I felt at the time.

I couldn't believe it.

And I couldn't deal with it at the time.

So my body dealt with it the best way it could.

And it resulted in that black smoke.

Which became so invading inside my body.

That it became cancerous.

It was there to protect me.

Because I couldn't deal with it at the time.

My Mum dying and my new baby growing inside me.

It was too much to take.

But now I have made the link.

I don't need that black smoke anymore. 
It's not in my bones anymore.

Or in my blood.

My bones are healthy and clear.

And my blood has a beautiful thick substance.

My body is healthy.

Free of the black smoke.

And I choose to release it.

At that point, still while tapping, Pauline said that the black smoke was panicking and angry. The smoke was gathering at the exits, which were her toes and fingertips, and it didn't want to let anybody or anything out.

That black smoke is angry and panicking.

I allow it to leave.

I set it free with love.

It's free to go.

Then Pauline said that the smoke was confused.

The black smoke is confused.

I'll show it the way.

The way to go to leave my body.

With love and thanks.

I'm setting it free.

Pauline said it was becoming grey smoke, still with bits of black at the "gates." It was still grey in her body, but her bones were not touched by it, and she was now seeing her bones look like a yellow sun. After tapping with wording that focused on the "gates" still appearing black, they became grey, then a white circle, then a mist. Then Pauline saw an image of her mother and felt her mother's presence around her. She felt assurance from her mother that she would be okay. She started crying:

I can feel my Mum.

And she said I was okay.

Aren't Mums annoying.

To be right all the time.

With that, Pauline burst out laughing and said, "Yes, Mum was always right!"

My Mum said l'd be okay.

Who am I not to believe her?

She was always right then.

And she's still right now.

She can see my strong bones.

And my healthy clear blood.

I'm so happy my Mum made herself felt by me!

And is looking after me. 
At this point, Pauline looked so relaxed that Lutz reported, "I found myself staring at her, so I had to explain to her that her features had completely changed in an hour." Pauline replied that she was scared because she felt so relaxed. They closed the session with a focus on Pauline's fear:

Even though I'm scared because I feel so relaxed, I deeply love and accept myself.

I'm so scared.

I'm not used to feeling that relaxed.

And it's scary.

Even though I could get used to it again. [Pauline laughs]

I could be open to the possibility.

That maybe it could be the way I feel every day.

And find it normal.

I'm so relaxed now and free of pain.

It's such a wonderful feeling.

In addition to her cancer, another health challenge Pauline faced was epilepsy. Shortly after the second session, described above, Pauline had a seizure and was coughing blood, necessitating a brief hospital visit. When she came in for an emergency session three days after the second session, she was shaky, weak, and "full of pain." She rated a pain in her right shoulder, arm, and extending to her fingertips as an 8. A shooting pain in her left temple was a 10. After tapping both down to 0 , the focus went to her throat, which she described as being like having "shards of glass" in there. The chemotherapy and vomiting over the past three years had damaged her esophagus, and she also was not producing enough saliva, so her mouth and throat were constantly dry. Sprays that had been suggested did not help.

Several rounds of tapping on the throat pain and dryness brought the pain down to 0 , and she could feel saliva in her mouth, which she had not experienced since her cancer treatment began. Also, Pauline had been a singer and had not been able to sing since the treatments started. Lutz recounts, "She played a song on the stereo and was able to sing along with no trouble at all. She was flabbergasted." Another outcome of this session is that at one point, Pauline was afraid that another seizure was coming on. This sensing would typically result in anxiety about another attack that she could not stop. But with the tapping on the pain, the anxiety also went away, and the seizure did not happen. As it turned out, Pauline found that she was able to use tapping to head off future seizures.

The morning following the third session, Pauline reported that she went to bed with no pain, had the soundest sleep in a very long time, woke up with no pain, and was able to keep her breakfast in. But then a headache returned, which Pauline explained was recurrent due to the radiation treatments she had received on her head for the brain tumor. Her head was painful to the touch, her hair literally hurt, and she reported being in agony if she tried to wash her hair. At the start of the fourth session, her headache was a 10. After several rounds of tapping, Pauline suddenly stopped and said that her headache was gone completely. She touched her head cautiously at first and then more roughly. She even went as far as pulling the hair from her scalp, amazed that she could not feel any of the excruciating pain she was used to. She was excited by the idea that she would be able to wash her hair that night. 
In the next session, Pauline was again in extreme pain and began to fear that a seizure was coming. She started to shake, hyperventilate, and moved into extreme panic. Lutz had her tap on a single point, to keep her focused, and got permission to tap on other points herself. By the end of the session, Pauline was again pain-free. After tapping on "I am so grateful I averted the fit," Pauline fell into Lutz' arms and cried with relief.

After five sessions in which pain was the primary theme, there was a shift. Pauline was now regularly sleeping through the night without her sleep being interrupted by pain, she was able to eat full meals and hold them down, and the chronic headache did not return. At this point, tests showed that the cancer had not spread to Pauline's bones, which had been a major concern. Nonetheless, her oncologist said that if it were anyone else with her Hodgkin's and brain tumor, the estimate would still be that she had only 6 to 18 months to live. But she was so positive in her attitude that he could not really say. Pauline explained to him that she was receiving tapping treatments that were helping her tremendously. They were keeping her pain-free after her having discontinued the pain medication, allowing her to sleep well, to have enough saliva so her tongue was not sticking to the roof of her mouth, and she had even been able to stop an epileptic fit a few days earlier. Her doctor was skeptical, but the liaison nurse, who already knew about EFT, was delighted and encouraged her to keep doing the tapping.

Space does not allow a detailed recounting of the remaining 15 sessions, but these five give a glimpse into how acupoint tapping sessions are conducted. Only some of the critical points in the remaining treatment will be mentioned. While pain would again be a focus when it returned, by the sixth session, attention moved to the Hodgkin's-related lumps throughout Pauline's body. Some would appear every few days and then disappear, though she could "still feel them lurking under my skin." They were very uncomfortable, and some were quite painful. An example of the wording used during the tapping that session was "Even though I have this annoying lump on my cheek, I now choose to dissolve it."

After a round of tapping, the lump on Pauline's cheek had changed from being hard to being "mushy." After further tapping and creative wording, it was completely gone. Pauline reported that she was getting "freaked" by being able to feel the lump dissolving. Not missing a beat, Lutz addressed this with: "Even though I'm freaked because my lump is disappearing, I choose to accept that it's going to be gone for good." Again, the charge on the feeling faded. Then the focus turned to a "big, hard, angry lump" on her neck. After diminishing bit by bit, it was gone. Then the focus went to a twinned pair of lumps on the back of her neck. All this in a single session. But something else emerged during that session. While the practitioner often suggests the wordings, the client also introduces them when moved to do so. Pauline used these phrases for the twin lumps as she tapped from point to point: "These twin lumps; They have to go too; The other lumps are gone; They have to go too; Like all the bad things in my childhood."

Lutz had not explored Pauline's childhood in the early sessions since pain was such a dominant feature. But it quickly emerged that Pauline had been molested, had been farmed out to relatives from ages 5 to 13, and felt emotionally abused by her mother in many ways, including her having "poisoned my brother and sister against me." All this was worked into the tapping phrasing as the first of the twin lumps dissolved, and then the other. Subsequent sessions focused on lumps throughout her breasts and lymph nodes, which were extremely painful: "The pain is getting angry; The lumps are getting angry; They're angry because they don't want to leave." The imagery grew more vivid over subsequent sessions (e.g., "this painful ball of green hair that feels like it's being 
pulled by a black root") and the wording more nuanced as the emotional wounds of Pauline's past, along with other issues such as the fear of death, were addressed along with the focus on her cancer. On seeing Pauline immediately following the tenth session, a month after their first tapping session, Pauline's husband "commented straightaway that her shoulders were not hunched up in pain anymore and her face had lost 10 years."

\subsection{Follow-Up}

Over the next nine months, the sessions produced marked progress as well as a few painful setbacks. After the first two months of treatment, the sessions averaged about one each month. Lutz' last entry in the case report, which described their $20^{\text {th }}$ session, 10 months after the first, relates that Pauline said, "the doctors can't believe the results, which are $65 \%$ better than what they expected." Her white blood cell count was increasing. She had put on weight. The doctors said to her, "You are a success!" Pauline "feels she's been given a second chance. She uses EFT every night when she's in pain, which still happens because of the ongoing treatment as well as hairline fractures brought on by the chemo. But her mind is in a very different place. She's trusting very much in her future. She can't wait for her son's communion in a few months' time, and she still remembers her fears of not being able to attend."

Upon inquiry about the accuracy of this report (which summarizes the 56 single-spaced posted pages), Lutz mentioned, "Pauline is alive and kicking as we speak, and it is wonderful to see. We are still in touch. She says EFT has literally saved her life" (e-mail communication, December 3, 2020). This was more than 11 years after their initial session, when Pauline had been told her life expectancy was between 6 and 18 months. Of course not every patient responds as rapidly and dramatically as did Pauline, and not every cancer patient who is treated with an energy psychology approach recovers, but the systematic clinical trials and additional case studies cited earlier suggest that the approach can have a strong positive impact on the course of an illness.

\section{Conclusion}

While utilizing the best medical interventions available remains the first line of treatment for serious illness, the potential value of working with the psychological aspects of disease is frequently underestimated. Energy psychology protocols have been used for managing mental states that impede immune function and healing, exploring emotional influences that contribute to illness, and mobilizing inner resources that promote healing. Evidence presented in this paper has demonstrated that manually stimulating selected acupoints while verbalizing phrases that evoke pertinent images, emotions, and cognitions sends signals that counter brain activities which are detrimental to health and increase brain activities that promote health. The approach can be applied by psychotherapists who are already trained in these methods or can be readily acquired by psychotherapists and other healthcare professionals.

\section{Acknowledgments}

The author wishes to thank John Freedom, Shoshana Garfield, Phil Mollon, and Lori Hops for their suggestions on earlier drafts of this paper. 


\section{Author Contributions}

The author conducted the background research for this study.

\section{Competing Interests}

The author conducts trainings, provides clinical services, and has written books related to the approach examined in this paper.

\section{References}

1. Nutton V. Ancient medicine. 2nd ed. New York: Routledge; 2013.

2. Lipton BH. The biology of belief. 10th ed. Carlsbad, CA: Hay House; 2016.

3. Llewellyn C, Ayers S, McManus IC, Newman SP, Petrie K, Revenson T, et al. Cambridge handbook of psychology, health and medicine. Cambridge: Cambridge University Press; 2019.

4. Cannon WB. The role of emotion in disease. Ann Intern Med. 1936; 9: 1453-1465.

5. Cannon WB. The wisdom of the body. Rev ed. New York: W.W. Norton; 1963.

6. Alexander F. Psychosomatic medicine. New York: W.W. Norton; 1950.

7. Levenson JL. Textbook of psychosomatic medicine and consultation-liaison psychiatry. 3rd ed. Washington, DC: American Psychiatric Association Publishing; 2019.

8. Straub RO. Health psychology: A biopsychosocial approach. 6th ed. New York: Worth Publishers; 2019.

9. Segerstrom S. The Oxford handbook of psychoneuroimmunology. New York: Oxford University Press; 2016.

10. Rakel D. Integrative medicine. 4th ed. Philadelphia: Elsevier; 2017.

11. Pert CB. Molecules of emotion: The science behind mind-body medicine. New York: Simon \& Schuster; 1999.

12. Scaer R. The body bears the burden: Trauma, dissociation, and disease. 3rd ed. New York: Routledge; 2014.

13. Seaward BL. Managing stress: Principles and strategies for health and well-being. 9th ed. Burlington, MA: Jones \& Bartlett; 2017.

14. Marlock G, Weiss $H$, Young $C$, Soth $M$. The handbook of body psychotherapy and somatic psychology. Berkeley, CA: North Atlantic Books; 2015.

15. Payne P, Levine PA, Crane-Godreau MA. Somatic experiencing: Using interoception and proprioception as core elements of trauma therapy. Front Psychol. 2015; 6: 93.

16. Gene-Cos N, Fisher J, Ogden P, Cantrell A. Sensorimotor psychotherapy group therapy in the treatment of complex PTSD. Ann Psychiatry Ment Health. 2016; 4: 1080.

17. Feinstein D. Energy psychology: Efficacy, speed, mechanisms. Explore. 2019; 15: 340-351.

18. Feinstein D. A survey of energy psychology practitioners: Who they are, what they do, who they help. Energy Psychol Theory Res Treat. 2016; 8: 33-39.

19. Feinstein D. Six empirically-supported premises about energy psychology: Mounting evidence for a controversial therapy. Advances in Mind-Body Medicine.

20. Hui KK, Liu J, Marina O, Napadow V, Haselgrove C, Kwong KK, et al. The integrated response of the human cerebro-cerebellar and limbic systems to acupuncture stimulation at ST 36 as evidenced by fMRI. Neuroimage. 2005; 27: 479-496. 
21. Fang J, Jin Z, Wang Y, Li K, Kong J, Nixon EE, et al. The salient characteristics of the central effects of acupuncture needling: Limbic-paralimbic-neocortical network modulation. Hum Brain Mapp. 2009; 30: 1196-1206.

22. Bagriantsev SN, Gracheva EO, Gallagher PG. Piezo proteins: Regulators of mechanosensation and other cellular processes. J Biol Chem. 2014; 289: 31673-31681.

23. Langevin HM, Yandow JA. Relationship of acupuncture points and meridians to connective tissue planes. Anat Rec. 2002; 269: 257-265.

24. Clond M. Emotional Freedom Techniques for anxiety: A systematic review with meta-analysis. J Nerv Ment Dis. 2016; 204: 388-395.

25. Nelms JA, Castel L. A systematic review and meta-analysis of randomized and nonrandomized trials of clinical Emotional Freedom Techniques (EFT) for the treatment of depression. Explore. 2016; 12: 416-426.

26. Sebastian B, Nelms J. The effectiveness of Emotional Freedom Techniques in the treatment of posttraumatic stress disorder: A meta-analysis. Explore. 2017; 13: 16-25.

27. David D, Cristea I, Hofmann SG. Why cognitive behavioral therapy is the current gold standard of psychotherapy. Front Psychiatry. 2018; 9: 4.

28. Brown RC, Witt A, Fegert JM, Keller F, Rassenhofer M, Plener PL. Psychosocial interventions for children and adolescents after man-made and natural disasters: A meta-analysis and systematic review. Psychol Med. 2017; 47: 1893-1905.

29. Mavranezouli I, Megnin-Viggars O, Daly C, Dias S, Stockton S, Meiser-Stedman R, et al. Research review: Psychological and psychosocial treatments for children and young people with posttraumatic stress disorder: A network meta-analysis. J Child Psychol Psychiatry. 2020; 61: 18-29.

30. Benor DJ, Ledger K, Toussaint L, Hett G, Zaccaro D. Pilot study of Emotional Freedom Techniques, wholistic hybrid derived from eye movement desensitization and reprocessing and Emotional Freedom Technique, and cognitive behavioral therapy for treatment of test anxiety in university students. Explore. 2009; 5: 338-340.

31. Gaesser AH, Karan OC. A randomized controlled comparison of Emotional Freedom Technique and cognitive-behavioral therapy to reduce adolescent anxiety: A pilot study. J Altern Complement Med. 2017; 23: 102-108.

32. Irgens AC, Hoffart A, Nysæter TE, Haaland $\vee \varnothing$, Borge FM, Pripp AH, et al. Thought field therapy compared to cognitive behavioral therapy and wait-list for agoraphobia: A randomized, controlled study with a 12-month follow-up. Front Psychol. 2017; 8: 1027.

33. Baker AH, Siegel MA. Emotional Freedom Techniques (EFT) reduces intense fears: A partial replication and extension of Wells et al. Energy Psychol Theory Res Treat. 2010; 2: 13-30.

34. Wells S, Polglase K, Andrews HB, Carrington P, Baker AH. Evaluation of a meridian-based intervention, Emotional Freedom Techniques (EFT), for reducing specific phobias of small animals. J Clin Psychol. 2003; 59: 943-966.

35. Lambrou P, Pratt G, Chevalier G. Physiological and psychological effects of a mind/body therapy on claustrophobia. Subtle Energ Energy Med J Arch. 2003; 14: 239-251.

36. Salas $M$, Brooks AJ, Rowe JE. The immediate effect of a brief energy psychology intervention (EFT) on specific phobias: A randomized controlled trial. Explore. 2011; 7: 155-161.

37. Church D. Clinical EFT (Emotional Freedom Techniques) as single session therapy: Cases, research, indications, and cautions. In Capturing the moment: Single session therapy and walkin service. Bethel, CT: Crown House; 2013. pp.125-150. 
38. Church D, Nelms J. Pain, range of motion, and psychological symptoms in a population with frozen shoulder: A randomized controlled dismantling study of clinical EFT (Emotional Freedom Techniques). Arch Sci Psychol. 2016; 4: 38-48.

39. Karatzias T, Power K, Brown K, McGoldrick T, Begum M, Young J, et al. A controlled comparison of the effectiveness and efficiency of two psychological therapies for posttraumatic stress disorder: Eye movement desensitization and reprocessing vs. Emotional Freedom Techniques. J Nerv Ment Dis. 2011; 199: 372-378.

40. Church D, Hawk C, Brooks A, Toukolehto O, Wren M, Dinter I, et al. Psychological trauma in veterans using EFT (Emotional Freedom Techniques): A randomized controlled trial. J Nerv Ment Dis. 2013; 201: 153-160.

41. Geronilla L, Minewiser L, Mollon P, McWilliams M, Clond M. EFT (Emotional Freedom Techniques) remediates PTSD and psychological symptoms in veterans: A randomized controlled replication trial. Energy Psychol Theory Res Treat. 2016; 8: 29-41.

42. American Psychological Association. Clinical practice guideline for the treatment of posttraumatic stress disorder (PTSD) in adults [Internet]. Washington, DC: American Psychological Association; 2017 [cited 2020 November 29th]. Available from: https://www.apa.org/ptsd-guideline/ptsd.pdf.

43. American Psychological Association. Cognitive therapy [Internet]. Washington, DC: American Psychological Association; 2017 [cited 2020 November 29th]. Available from: https://www.apa.org/ptsd-guideline/treatments/cognitive-therapy.

44. Steenkamp MM, Litz BT, Hoge CW, Marmar CR. Psychotherapy for military-related PTSD: A review of randomized clinical trials. JAMA. 2015; 314: 489-500.

45. Church D, Yount G, Rachlin K, Fox L, Nelms J. Epigenetic effects of PTSD remediation in veterans using clinical EFT (Emotional Freedom Techniques): A randomized controlled trial. Am J Health Promot. 2018; 32: 112-122.

46. Maharaj ME. Differential gene expression after Emotional Freedom Techniques (EFT) treatment: A novel pilot protocol for salivary mRNA assessment. Energy Psychol Theory Res Treat. 2016; 8: 17-32.

47. Church D, Yount G, Brooks AJ. The effect of Emotional Freedom Techniques (EFT) on stress biochemistry: A randomized controlled trial. J Nerv Ment Dis. 2012; 200: 891-896.

48. Stapleton P, Crighton G, Sabot D, O'Neill HM. Reexamining the effect of emotional freedom techniques on stress biochemistry: A randomized controlled trial. Psychol Trauma. 2020; 12: 869-877.

49. Swingle P. Emotional Freedom Techniques (EFT) as an effective adjunctive treatment in the neurotherapeutic treatment of seizure disorders. Energy Psychol Theory Res Treat. 2010; 2: 2938.

50. Stapleton P, Buchan C, Mitchell I, McGrath J, Gorton P, Carter B. An initial investigation of neural changes in overweight adults with food cravings after Emotional Freedom Techniques. OBM Integr Complemen Med. 2019; 4. Doi: 10.21926/obm.icm.1901010.

51. Lightbody ST. 361 classical acupuncture points: The names, functions, descriptions and locations. New Jersey: World Scientific; 2020.

52. Ahn AC, Colbert AC, Anderson BJ, Martinsen OG, Hammerschlag R, Cina S, et al. Electrical properties of acupuncture points and meridians: A systematic review. Bioelectromagnetics. 2008; 29: 245-256. 
53. Li J, Wang $\mathrm{Q}$, Liang $\mathrm{H}$, Dong $\mathrm{H}$, Li $\mathrm{Y}, \mathrm{Ng} \mathrm{E}$, et al. Biophysical characteristics of meridians and acupoints: A systematic review. Evid Based Complementary Altern Med. 2012; 2012: 793841.

54. McDonald J, Janz S. The acupuncture evidence project: A comparative literature review [Internet]. Brisbane: Australian Acupuncture and Chinese Medicine Association; 2017 [cited 2021 February 1st]. Available from: https://www.asacu.org/wpcontent/uploads/2017/09/Acupuncture-Evidence-Project-The.pdf.

55. Takakura N, Yajima H. Analgesic effect of acupuncture needle penetration: A double-blind crossover study. Open Med. 2009; 3: e54-e61.

56. Church D. The EFT manual. 4th ed. Fulton, CA: Energy Psychology Press; 2018.

57. Henningsen $P$, Zimmermann $T$, Sattel $H$. Medically unexplained physical symptoms, anxiety, and depression: A meta-analytic review. Psychosom Med. 2003; 65: 528-533.

58. Katon W, Lin EH, Kroenke K. The association of depression and anxiety with medical symptom burden in patients with chronic medical illness. Gen Hosp Psychiatry. 2007; 29: 147-155.

59. Masih J, Belschak F, Verbeke JW. Mood configurations and their relationship to immune system responses: Exploring the relationship between moods, immune system responses, thyroid hormones, and social support. PloS ONE. 2019; 14: e0216232.

60. Thomas R, Cutinho S, Aranha D. Emotional Freedom Techniques (EFT) reduces anxiety among women undergoing surgery. Energy Psychol. 2017; 9: 18-25.

61. Cartland A. Emotional Freedom Techniques (EFT) remediates dental fear: A case series. Energy Psychol. 2016; 8: 42-66.

62. Bach D, Groesbeck G, Stapleton P, Sims R, Blickheuser K, Church D. Clinical EFT (Emotional Freedom Techniques) improves multiple physiological markers of health. J Evid Based Integr Altern Med. 2019; 24: 2515690X18823691.

63. Kalla $M$, Simmons $M$, Robinson A, Stapleton P. Emotional Freedom Techniques (EFT) as a practice for supporting chronic disease healthcare: A practitioners' perspective. Disabil Rehabil. 2018; 40: 1654-1662.

64. Kalla M, Simmons M, Robinson A, Stapleton P. Making sense of chronic disease using Emotional Freedom Techniques (EFT): An existential view of illness. Explore. 2020; 16: 214-224.

65. Brattberg G. Self-administered EFT in individuals with fibromyalgia: A randomized trial. Integr Med. 2008; 7: 30-35.

66. Church D, Stapleton P, Sabot D. App-based delivery of clinical Emotional Freedom Techniques: Cross-sectional study of app user self-ratings. JMIR mhealth uhealth. 2020; 8: e18545.

67. Min MO, Minnes S, Kim H, Singer LT. Pathways linking childhood maltreatment and adult physical health. Child Abuse Negl. 2013; 37: 361-373.

68. Chang $X$, Jiang $X$, Mkandarwire $T$, Shen $M$. Associations between adverse childhood experiences and health outcomes in adults aged 18-59 years. PLoS ONE. 2019; 14: e0211850.

69. Di Rienzo F, Saruco E, Church D, Daligault S, Delpuech C, Gurret JM, et al. Neuropsychological correlates of an energy psychology intervention on flight phobia: A MEG single-case study. 2019. Doi: 10.31234/osf.io/s3hce.

70. Wittfoth $D$, Pfeiffer $A$, Bohne $M$, Lanfermann $H$, Wittfoth $M$. Emotion regulation through bifocal processing of fear inducing and disgust inducing stimuli. BMC Neurosci. 2020; 21: 47.

71. Eden D. Energy medicine. New York: Tarcher/Penguin Random House; 2008.

72. Church D. Bliss brain: The neuroscience of remodeling your brain for resilience. Carlsbad, CA: Hay House; 2020. 
73. Babamahmoodi A, Arefnasab Z, Noorbala AA, Ghanei M, Babamahmoodie F, Alipour A, et al. Emotional Freedom Technique (EFT) effects on psychoimmunological factors of chemically pulmonary injured veterans. Iran J Allergy Asthma Immunol. 2015; 14: 37-47.

74. Baker BS, Hoffman CJ. Emotional Freedom Techniques (EFT) to reduce the side effects associated with tamoxifen and aromatase inhibitor use in women with breast cancer: A service evaluation. Eur J Integr Med. 2015; 7: 136-142.

75. Bougea AM, Spandideas N, Alexopoulos EC, Thomaides T, Chrousos GP, Darviri C. Effect of the Emotional Freedom Technique on perceived stress, quality of life, and cortisol salivary levels in tension-type headache sufferers: A randomized controlled trial. Explore. 2013; 9: 91-99.

76. Hajloo $M$, Ahadi $H$, Rezabakhsh $H$, Mojembari AK. Investigation on Emotional-Freedom Technique effectiveness in diabetic patients' blood sugar control. Mediterr J Soc Sci. 2014; 5: 1280.

77. Feinstein D. Words to tap by: The uses of language in energy psychology protocols. Energy Psychol Theory Res Treat. 2019; 11: 41-56.

78. Lee MS, Ernst E. Acupuncture for pain: An overview of Cochrane reviews. Chin J Integr Med. 2011; 17: 187-189.

79. Stapleton P, Chatwin H, Shepperd L, McSwan J. The lived experience of chronic pain and the impact of brief Emotional Freedom Techniques (EFT) group therapy on coping. Energy Psychol Theory Res Treat. 2016; 8: 18-28.

80. Ortner $\mathrm{N}$. The tapping solution for pain relief: A step-by-step guide to reducing and eliminating chronic pain. Carlsbad, CA: Hay House; 2016.

81. Wolpe J. Psychotherapy by reciprocal inhibition. Stanford, CA: Stanford University Press; 1958.

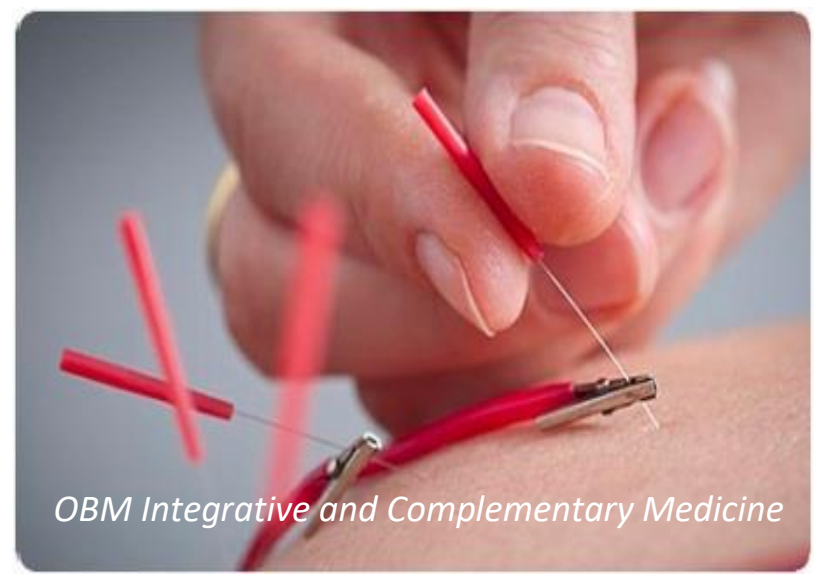

Enjoy OBM Integrative and Complementary Medicine by:

1. Submitting a manuscript

2. Joining in volunteer reviewer bank

3. Joining Editorial Board

4. Guest editing a special issue

For more details, please visit: http://www.lidsen.com/journals/icm 